\title{
INFLUÊNCIA DO SISTEMA DE PLANTIO SOBRE ATRIBUTOS QUÍMICOS DE SUBSTRATO DE CAVA DE EXTRAÇÃO DE ARGILA
}

\author{
Anderson Ribeiro Santiago ${ }^{1}$, Deborah Guerra Barroso ${ }^{2}$, Andrea Vita Reis Mendonça ${ }^{3}$, Teresa Aparecida \\ Soares Freitas ${ }^{3}$, Kelly Ribeiro Lamônica ${ }^{4}$, José Geraldo Araújo Carneiro ${ }^{5}$ \\ ${ }^{1}$ Eng. Florestal, M.Sc., NATURATINS, Palmas, TO, Brasil - asantiago@ naturatins.to.gov.br \\ ${ }^{2}$ Eng $^{\mathrm{a}}$. Agrônoma, Dra ., UENF, Campos dos Goytacazes, RJ, Brasil - deborah@uenf.br \\ ${ }^{3}$ Eng $^{\mathrm{a}}$. Florestal, Dr ${ }^{\mathrm{a}}$., UFRB, Cruz das Almas, BA, Brasil - avrmendonca@ @otmail.com - tas_freitas@hotmail.com \\ ${ }^{4}$ Eng. ${ }^{a}$ Agrônoma, M.Sc., Doutoranda UENF, Campos dos Goytacazes, RJ, Brasil - krlamonica@ gmail.com \\ ${ }^{5}$ Eng. Florestal, Ph.D., UENF, Campos dos Goytacazes, RJ, Brasil - carneiro@uenf.br \\ Recebido para publicação: 15/10/2007 - Aceito para publicação: 28/10/2008
}

\begin{abstract}
Resumo
A diminuição gradativa nos teores de matéria orgânica e nutrientes em plantios comerciais de eucalipto tem sido apontada como fator negativo na sustentabilidade ecológica desses empreendimentos. $\mathrm{O}$ objetivo deste trabalho foi avaliar o efeito de sistemas de plantio de eucalipto (monocultivos e consorciados) nos atributos químicos de uma cava de extração de argila. Para isso, implantaram-se dois experimentos, um constituído por Eucalyptus camaldulensis, E. tereticornis, E. robusta e E. pellita em monocultivo, e outro com as mesmas espécies de eucalipto consorciadas com Sesbania virgata, sendo a leguminosa manejada sob podas periódicas. Os dois experimentos foram conduzidos segundo um delineamento em blocos casualizados com 4 repetições. Foram coletadas amostras de substrato, antes e um ano após a implantação dos eucaliptos, nas profundidades 0-20 e 20-40 cm. Para análise do efeito dos sistemas de plantio nas características químicas do substrato, foi aplicado o teste t para dados não pareados, com variâncias populacionais desconhecidas. Com base nos resultados, foi possível constatar que os plantios consorciados reduziram de maneira mais acentuada os teores de Soma de Bases (SB) e Capacidade de Troca Catiônica efetiva (t) em relação aos monocultivos. Entretanto, nos tratamentos que continham a espécie E. Tereticornis não houve alteração dos teores de nutrientes, da SB e do valor t do substrato. Os teores de matéria orgânica não decresceram em nenhum dos plantios.

Palavras-chave: Plantio puro; plantio consorciado; área degradada.
\end{abstract}

\begin{abstract}
Influence of planting systems on the chemical characteristic of substratum of clay mining digging. The decrease in the organic matter and nutrients contents in eucalyptus plantings has been the negative factor in the ecological sustainability of those areas. The objective of this work was to evaluate the effect of planting systems (pure and mixed) in the chemical attributes of clay mining digging. Were two experiments implanted, one constituted by Eucalyptus camaldulensis, E. tereticornis, E. robusta. and E. pellita in pure plantings, and other with the same eucalyptus species mixed with Sesbania virgata. The two experiments were carried out in Randomized block design with 4 replications. Substratum samples were collected, before and one year eucalyptus out planting, at 0 20 and $20-40 \mathrm{~cm}$ depths. For analysis of the planting system effects on the chemical characteristics of the substratum was applied the $t$ test. The mixed plantings reduced a lot the sum of bases (SB) and cation exchange capacity (CEC) in relation to the pure plantings. However, in the treatments with $E$. tereticornis there was no alteration of the nutrient contents, of SB and CEC of the substratum. The organic matter did not decrease in none of the plantings.

Keywords: Pure planting; mixed planting; disturbed land.
\end{abstract}

\section{INTRODUÇÃO}

A atividade de mineração altera as características químicas, físicas e microbiológicas dos solos, impondo limitações ao estabelecimento e desenvolvimento espontâneo de cobertura vegetal (DIONÍSIO 
et al., 1994; RUIVO et al., 2001; VALICHESKI, 2004). Entre as maiores modificações nos solos provocadas pela extração mineral, destacam-se a perda de estruturação e a diminuição de teores de matéria orgânica e de nutrientes (SOUZA et al., 2001). Em processos de mineração de superfície, como a exploração de argila, ocorre a retirada das camadas de solo estruturadas, chegando a expor o lençol freático e formando, em alguns locais, condições de elevada salinidade, conforme observado por Valicheski (2004) em Campos dos Goytacazes (RJ).

Estudos têm demonstrado que o plantio de espécies arbóreas, em consórcio ou não, pode alterar de forma benéfica alguns atributos químicos e físicos dos solos (GACHENGO et al., 1999; VEZZANI et al., 2001; MARTINS et al., 2002). O cultivo em aleias, onde árvores são podadas periodicamente, funcionando como espécies adubadeiras, também tem permitido melhoria nas características químicas do solo, pela reciclagem mais eficiente dos nutrientes a partir da biomassa resultante das podas (HAGGAR et al., 1993; MAFRA et al., 1998a, MAFRA et al., 1998b).

Parrota (1999), consorciando Casuarina equisetifolia L., Eucalyptus robusta Sm. e Leucaena leucocephala (Lam.) de Wit, constatou maior estoque de nutrientes ( $, \mathrm{P}, \mathrm{K}, \mathrm{Ca}$ e $\mathrm{Mg}$ ) nos primeiros $40 \mathrm{~cm}$ de solo em relação ao plantio puro das mesmas espécies. No consórcio de Tectona grandis L.f. com Leucaena leucocephala (Lam.) de Wit, Kumar (1998) verificou aumento na disponibilidade de $\mathrm{N}$ e P no solo. Vezzani et al. (2001), consorciando Eucalyptus grandis Hill ex Maiden com Acacia mearnsii de Wild., verificaram aumentos de $\mathrm{pH}$ e diminuição das concentrações de $\mathrm{Al}$ trocável nos primeiros $20 \mathrm{~cm}$ de solo em relação ao monocultivo de eucalipto.

Num cultivo em aleias, Mafra et al. (1998b), constataram que as podas na espécie arbórea proporcionaram aumentos significativos no teor de carbono orgânico da camada arável do solo. Nesse trabalho, uma única espécie arbórea utilizada no sistema agroflorestal, a leucena, contribuiu com cerca de 70 a $85 \%$ da adição total de $\mathrm{N}, \mathrm{P}, \mathrm{Ca}$ e $\mathrm{Mg}$, o equivalente, segundo os autores, a uma adubação de $280 \mathrm{~kg} . \mathrm{ha}^{-1}$ de ureia, $30 \mathrm{~kg} . \mathrm{ha}^{-1}$ de superfosfato simples e $100 \mathrm{~kg}^{-h^{-1}}$ de cloreto de potássio.

Sendo a revegetação considerada parte essencial no processo de recuperação de áreas degradadas pela mineração (SOUZA et al., 2001), o desafio é determinar o melhor manejo e encontrar espécies que se adaptem a essas áreas e sejam ainda capazes de melhorar as condições edáficas.

O objetivo deste trabalho foi avaliar a influência dos sistemas de plantio, monocultivo e consorciado, de algumas espécies de eucalipto com Sesbania virgata (Cav.) Pers, sob manejo de podas periódicas da leguminosa, nos atributos químicos de uma cava de extração de argila.

\section{MATERIAL E MÉTODOS}

Em uma cava de extração de argila, localizada no distrito de Campo Limpo, Campos dos Goytacazes, RJ ( $21^{\circ} 51$ 'S, $41^{\circ} 14^{\prime} \mathrm{W}$, a $14 \mathrm{~m}$ de altitude), foram instalados dois experimentos, sendo um cultivado em monocultivo, com as espécies de eucalipto (Eucalyptus camaldulensis Dehn, E. tereticornis Sm., E. robusta Sm. e E. pellita F. Muell), e outro em sistema de cultivo consorciado com cada uma dessas espécies plantadas nas entrelinhas de Sesbania virgata (Cav.) Pers.

A extração de argila é realizada, no município, em planície com material do período Quaternário, do Baixo Paraíba do Sul, local em que os solos apresentam grandes reservas de argila. Em trabalho de caracterização edáfica da área, Valicheski (2004) identificou o solo original como sendo um Cambissolo Háplico Sódico gleico salino, formado a partir de sedimentos aluviais de uma sequência variada de extratos do rio Paraíba do Sul, ocorrida no período Quaternário (Holoceno). O relevo apresenta-se plano. Nos últimos 10 anos, a temperatura média mensal do município variou entre $21,4{ }^{\circ} \mathrm{C}$ e $27,7{ }^{\circ} \mathrm{C}$ e a precipitação média anual foi de $852 \mathrm{~mm}$, com chuvas concentradas no período de outubro a dezembro. No período experimental, a temperatura média mensal variou de $18,3{ }^{\circ} \mathrm{C}$ a $28,2{ }^{\circ} \mathrm{C}$, com precipitação de $767 \mathrm{~mm}$. O município tem histórico de déficit hídrico.

A cava tem um hectare de extensão e foi explorada no período de 1999 a 2002, a uma profundidade de extração variando de 2 a $2,5 \mathrm{~m}$. Assim, no período experimental, o lençol freático foi encontrado, no período seco, a 1,4 m de profundidade, e no período chuvoso, a $1 \mathrm{~m}$.

Os dois experimentos (monocultivo e consorciado) foram conduzidos segundo delineamento em blocos casualizados com 4 repetições. Cada experimento foi delineado em 4 tratamentos, constituído pelas espécies de eucalipto, sendo cada parcela composta por 18 plantas, sem bordadura. O espaçamento de plantio nos dois experimentos foi de $3 \times 2 \mathrm{~m}$, com covas medindo $20 \times 20 \times 20 \mathrm{~cm}$, sem realização de 
adubação, em função das análises químicas do substrato (Tabela 1). A leguminosa arbustiva, Sesbania virgata (Cav.) Pers, na época do plantio dos eucaliptos, estava com altura média de 2,80 $\pm 0,12 \mathrm{~m}$ e 1 ano de idade e recebeu três podas, sendo a primeira aos 10 meses (em maio de 2003), antes do plantio dos eucaliptos, a segunda aos 16 (em novembro de 2003) e a terceira aos 22 meses (em maio de 2004).

O material resultante da poda da Sesbania foi distribuído, de forma homogênea, nas entrelinhas de plantio. As duas primeiras podas foram realizadas apenas para uniformização. Na terceira poda foram selecionadas duas plantas médias por parcela e foi quantificada a biomassa resultante da poda. Para isso, o material podado foi coletado antes de sua distribuição e devidamente separado em folhas, galhos e frutos.

Os diferentes componentes da parte aérea da Sesbania foram pesados após o corte, obtendo-se o peso de massa fresca. A estimativa da massa seca dos componentes resultantes da poda foi realizada retirando-se amostras deste material, que foram pesadas no campo e levadas à estufa de circulação forçada a $65^{\circ} \mathrm{C}$, até peso constante, conforme Freitas (2003).

Após a secagem, o material vegetal foi pesado, moído e submetido à digestão nítrico-perclórica e sulfúrica, no caso do N, para determinação dos teores de nutrientes nos galhos, folhas e frutos. Foram determinados os teores de $\mathrm{N}$ (Nessler), P (colorimetria), K (fotometria de chama) e C (digestão com dicromato de potássio), além dos teores de micronutrientes ( $\mathrm{Fe}, \mathrm{Cu}, \mathrm{Zn}$ e $\mathrm{Mn})$, segundo Jones Jr. et al. (1991) e Malavolta et al. (1997).

A amostragem do substrato da cava para análises químicas foi realizada em duas épocas (julho de 2003, antes da implantação dos eucaliptos, e julho de 2004, após a implantação dos eucaliptos) e duas profundidades ( 0 a $20 \mathrm{~cm}$ e 20 a $40 \mathrm{~cm}$ ), sendo a primeira para fins de caracterização e a segunda para avaliação do efeito dos sistemas de plantio e da poda da Sesbania. Foram determinados pH (em água), acidez potencial, condutividade elétrica da pasta saturada e carbono orgânico e os teores de $\mathrm{P}, \mathrm{K}$ e $\mathrm{Na}$ extraíveis por Mehlich ${ }^{-1}$; $\mathrm{Ca}, \mathrm{Mg}$ e Al trocáveis pelo $\mathrm{KCl} 1 \mathrm{~mol} \mathrm{~L}^{-1}$, segundo metodologia descrita pela EMBRAPA (1997).

As análises físicas da densidade do substrato (anel volumétrico) e da partícula (balão volumétrico), da porosidade total, macro e microporosidade (mesa de tensão, com amostras saturadas) e da composição granulométrica, pelo método da pipeta, foram feitas em julho de 2003 apenas para caracterização inicial.

Em cada repetição foi coletada uma amostra, composta por quatro subamostras, em duas profundidades. Para análise granulométrica e caracterização química, as profundidades de amostragem foram 0 a $20 \mathrm{~cm}$ e 20 a $40 \mathrm{~cm}$. Para porosidade, densidade da partícula e do substrato, as profundidades de amostragem foram 0 a $10 \mathrm{~cm}$ e 10 a $20 \mathrm{~cm}$.

Para análise do material vegetal resultante da poda e caracterização química e física do substrato, foi aplicado o teste de Lilliefors $(\alpha=0,01)$ para se verificar se os dados apresentaram distribuição normal (CAMPOS, 1979). Quando eles apresentaram distribuição normal, foi obtido um intervalo de confiança para a média $(\alpha=0,01)$. Para análise do efeito dos sistemas de plantio nas características químicas do substrato, foi aplicado o teste t para dados não pareados, com variâncias populacionais desconhecidas $(\alpha=0,05)$.

\section{RESULTADOS E DISCUSSÃO}

Os atributos químicos do substrato (Tabela 1), no momento da caracterização, encontravam-se em níveis adequados para a cultura do eucalipto, exceto para o P (BARROS et al., 2000; NOVAIS et al., 1986). No entanto, para alguns atributos físicos (tabelas 2 e 3), como macroporosidade, os valores determinados foram muito baixos (Tabela 2), semelhantes aos encontrados por Valicheski et al. (2003) $\left(0,18\right.$ e $\left.0,23 \mathrm{~m}^{3} \cdot \mathrm{m}^{-3}\right)$ em outras cavas de extração de argila. Moraes et al. (2002) relataram que valores baixos de macroporos são reflexo da perda da estrutura original do solo, o que resulta na redução da aeração e da movimentação de água no solo, podendo dificultar o desenvolvimento do sistema radicular (KIEHL, 1979).

Quanto ao efeito dos sistemas de plantio e do manejo de podas da Sesbania nas características químicas das cavas, pode-se observar uma diminuição entre o que havia antes e o que foi encontrado após 12 meses do plantio dos eucaliptos (Tabela 4). Houve, para alguns tratamentos, uma redução nos teores de $\mathrm{Ca}$ e $\mathrm{Mg}$ trocáveis e de fósforo no substrato da cava, e isso foi mais frequente nos plantios 
consorciados (Tabela 4). Os teores de potássio no substrato mantiveram-se com valores próximos aos encontrados antes do plantio dos eucaliptos (Tabela 4).

Tabela 1. Caracterização química do substrato da cava resultante da extração de argila.

Table 1. Chemical characterization of the digging substratum, resulting from the clay mining, before eucalyptus planting.

\begin{tabular}{|c|c|c|c|c|c|c|c|c|c|c|c|c|}
\hline \multicolumn{13}{|c|}{ Plantio puro } \\
\hline \multirow{2}{*}{$\begin{array}{l}\text { Prof. } \\
\text { (cm) }\end{array}$} & \multirow{2}{*}{$\begin{array}{c}\mathbf{p H} \\
\left(\mathbf{H}_{2} \mathbf{O}\right)\end{array}$} & \multirow{2}{*}{$\begin{array}{c}P \\
\left(\mathbf{m g . d m}{ }^{-3}\right)\end{array}$} & \multirow{2}{*}{$\underset{\left(\text { g.dm }^{-3}\right)}{\text { MO }}$} & $\mathrm{Na}$ & $\mathbf{C a}$ & Mg & $\mathbf{H}+\mathbf{A l}$ & $\mathbf{K}$ & $\mathbf{T}$ & SB & \multirow{2}{*}{$\begin{array}{c}\mathbf{V} \\
(\%)\end{array}$} & \multirow{2}{*}{$\underset{\left(\mathbf{d S} . \mathbf{m}^{-1}\right)}{\mathbf{C E}_{\text {es }}}$} \\
\hline & & & & \multicolumn{7}{|c|}{$\left(\mathrm{cmol}_{\mathrm{c}} \cdot \mathrm{dm}^{-3}\right)$} & & \\
\hline $0-20$ & $5,7 \pm 0,2$ & $26,4 \pm 33,2$ & $25,2 \pm 4,6$ & $1,2 \pm 0,3$ & $8,0 \pm 3,5$ & $4,8 \pm 0,6$ & $2,8 \pm 0,3$ & $0,2 \pm 0,0$ & $16,9 \pm 3,6$ & $14,1 \pm 3,6$ & $83,5 \pm 4,0$ & $1,4 \pm 0,7$ \\
\hline $\mathrm{CV} \%$ & 2,28 & 79,20 & 11,60 & 15,42 & 27,48 & 8,37 & 6,30 & 9,84 & 13,27 & 16,18 & 3,01 & 30,46 \\
\hline $20-40$ & $6,1 \pm 0,7$ & $13,9 \pm 11,4$ & $16,0 \pm 10,7$ & $1,7 \pm 0,9$ & $5,6 \pm 3,9$ & $3,3 \pm 1,8$ & $1,9 \pm 1,1$ & $0,1 \pm 0,0$ & $12,5 \pm 6,2$ & $10,6 \pm 5,2$ & $85,2 \pm 3,1$ & $1,5 \pm 1,5$ \\
\hline $\mathrm{CV} \%$ & 6,77 & 51,93 & 41,84 & 35,71 & 43,65 & 34,85 & 37,81 & 24,00 & 31,27 & 30,62 & 2,29 & 61,97 \\
\hline \multicolumn{13}{|c|}{ Plantio consorciado } \\
\hline \multirow{2}{*}{$\begin{array}{l}\text { Prof. } \\
\text { (cm) }\end{array}$} & \multirow{2}{*}{$\begin{array}{c}\mathbf{p H} \\
\left(\mathbf{H}_{2} \mathbf{O}\right)\end{array}$} & \multirow{2}{*}{$\underset{(\mathrm{mg.dm}}{P}$} & \multirow{2}{*}{$\underset{\left(\mathrm{g}^{\mathrm{d}} \mathrm{dm}^{-3}\right)}{\text { MO }}$} & $\mathrm{Na}$ & $\mathbf{C a}$ & Mg & $\mathbf{H}+\mathbf{A l}$ & $\mathbf{K}$ & $\mathbf{T}$ & SB & \multirow{2}{*}{$\underset{(\%)}{V}$} & \multirow{2}{*}{$\begin{array}{c}\mathrm{CE}_{\mathrm{es}} \\
\left(\mathrm{dS} . \mathrm{m}^{-1}\right)\end{array}$} \\
\hline & & & & \multicolumn{7}{|c|}{$\left(\mathrm{cmol}_{\left.\mathrm{c} \cdot \mathrm{dm}^{-3}\right)}\right.$} & & \\
\hline $0-20$ & $5,7 \pm 0,5$ & $18,6 \pm 16,0$ & $24,1 \pm 10,4$ & $1,2 \pm 1,2$ & $7,3 \pm 2,2$ & $5,0 \pm 1,1$ & $3,0 \pm 0,8$ & $0,1 \pm 0,0$ & $16,9 \pm 2,4$ & $13,9 \pm 2,3$ & $82,5 \pm 4,4$ & $1,3 \pm 0,5$ \\
\hline $\mathrm{CV} \%$ & 5,50 & 54,18 & 27,22 & 47,21 & 18,75 & 14,27 & 17,83 & 20,63 & 9,06 & 10,17 & 3,33 & 23,97 \\
\hline $20-40$ & $5,7 \pm 0,3$ & $16,8 \pm 14,7$ & $22,0 \pm 6,7$ & $1,9 \pm 0,8$ & $6,4 \pm 2,0$ & $4,6 \pm 0,2$ & $3,0 \pm 0,6$ & $0,1 \pm 0,0$ & $15,9 \pm 1,6$ & $13,1 \pm 1,9$ & $82,0 \pm 4,9$ & $1,5 \pm 0,4$ \\
\hline CV\% & 3,20 & 55,10 & 19,26 & 26,72 & 19,31 & 3,08 & 13,59 & 18,32 & 6,38 & 9,05 & 3,74 & 15,10 \\
\hline
\end{tabular}

Prof.: profundidade; MO: matéria orgânica; T: CTC pH 7,0; SB: soma de bases trocáveis; V: saturação por bases; $\mathrm{CE}_{\mathrm{es}}$ : condutividade elétrica da pasta saturada; $\mathrm{CV}$ : coeficiente de variação.

Extrator para $\mathrm{Na}, \mathrm{P}$ e K $-\mathrm{Mehlich}^{-1}$; Extrator para $\mathrm{Ca}^{+2} \mathrm{e} \mathrm{Mg}^{+2}-\mathrm{KCl} 1$ mol.L $\mathrm{L}^{-1}$; Extrator para $\mathrm{H}+\mathrm{Al}$ - Acetato de cálcio.

Tabela 2. Densidade do substrato ( $\rho$ ) e de partícula ( $\rho s)$, porosidade total (PT), macroporosidade (Mac.) e microporosidade (Mic.) do substrato da cava.

Table 2 Substratum density ( $\rho)$ and of particle ( $\rho$ s), total porosity (PT), macroporosity (Mac.) and microporosity (Mic.) of the digging clay mining substratum.

\begin{tabular}{|c|c|c|c|c|c|}
\hline \multicolumn{6}{|c|}{ Plantio puro } \\
\hline Prof. (cm) & $\rho\left(\mathrm{mg}^{\prime} \mathrm{m}^{-3}\right)$ & ps $\left(\mathrm{mg} . \mathrm{m}^{-3}\right)$ & $\operatorname{Mac}\left(\mathrm{m}^{3} \cdot \mathrm{m}^{-3}\right)$ & $\operatorname{Mic}\left(\mathrm{m}^{3} \cdot \mathrm{m}^{-3}\right)$ & PT $\left(\mathrm{m}^{3} \cdot \mathrm{m}^{-3}\right)$ \\
\hline $0-10$ & $1,21 \pm 0,25$ & $2,58 \pm 0,08$ & $0,01 \pm 0,03$ & $0,53 \pm 0,10$ & $0,53 \pm 0,09$ \\
\hline 10-20 & $1,22 \pm 0,10$ & $2,59 \pm 0,15$ & $0,00 \pm 0,01$ & $0,52 \pm 0,03$ & $0,53 \pm 0,03$ \\
\hline \multicolumn{6}{|c|}{ Plantio consorciado } \\
\hline Prof. (cm) & $\rho\left(\mathrm{mg} \cdot \mathrm{m}^{-3}\right)$ & ps $\left(\mathrm{mg} \cdot \mathrm{m}^{-3}\right)$ & $\operatorname{Mac}\left(m^{3} \cdot m^{-3}\right)$ & $\operatorname{Mic}\left(\mathrm{m}^{3} \cdot \mathrm{m}^{-3}\right)$ & PT $\left(\mathrm{m}^{3} \cdot \mathrm{m}^{-3}\right)$ \\
\hline $0-10$ & $1,19 \pm 0,03$ & $2,47 \pm 0,07$ & $0,08 \pm 0,22$ & $0,44 \pm 0,20$ & $0,52 \pm 0,02$ \\
\hline $10-20$ & $1,24 \pm 0,10$ & $2,53 \pm 0,17$ & $0,12 \pm 0,18$ & $0,38 \pm 0,21$ & $0,50 \pm 0,05$ \\
\hline
\end{tabular}

Na camada de 0 a $20 \mathrm{~cm}$ de profundidade, houve redução nos teores de $\mathrm{P}, \mathrm{Ca}$ e $\mathrm{Mg}$ no consórcio $S$. virgata com E. robusta (Tabela 4). Os teores de $\mathrm{Ca}$ e $\mathrm{Mg}$ também foram reduzidos no plantio consorciado de E. camaldulensis com S. virgata (Tabela 4). O E. pellita, no consórcio, resultou na redução apenas do $\mathrm{Mg}$ (Tabela 4). No monocultivo, o teor de $\mathrm{Mg}$ só não foi reduzido no plantio de $E$. tereticornis.

Apesar do tratamento com E. tereticornis, nos dois sistemas de plantio, não ter resultado na alteração da maioria dos teores de nutrientes avaliados, constatou-se redução no teor de $\mathrm{Mg}$ na camada de 20 a $40 \mathrm{~cm}$ de profundidade (Tabela 4).

Outro aspecto importante que deve ser mencionado é que os teores de $\mathrm{P}, \mathrm{K}$ e $\mathrm{Ca}$ em todos os monocultivos de eucalipto na camada de 20 a $40 \mathrm{~cm}$ de profundidade foram maiores em relação à avaliação inicial (Tabela 4).

A redução de $\mathrm{Ca}$ e $\mathrm{P}$ no substrato sob plantio consorciado não pode ser atribuída apenas à presença da Sesbania, pois isso não ocorre no consórcio com todas as espécies de eucalipto. Isso destaca as diferenças que podem ser obtidas nas diversas combinações de espécies. Como exemplo, observa-se 
que o consórcio de E. robusta com S. virgata potencializou a extração de $\mathrm{P}$ e $\mathrm{Mg}$ até mesmo na camada de 20 a $40 \mathrm{~cm}$ de profundidade (Tabela 4).

Tabela 3. Composição granulométrica ${ }^{1}$ do substrato da cava de extração de argila.

Table 3. Texture of the digging of clay mining substratum.

\begin{tabular}{lccc}
\hline \multicolumn{4}{c}{ Plantio puro } \\
\hline Prof. $(\mathbf{c m})$ & Areia (\%) & Silte (\%) & Argila (\%) \\
\hline $0-20$ & $12,40 \pm 12,12$ & $37,07 \pm 3,66$ & $50,52 \pm 10,90$ \\
$20-40$ & $34,17 \pm 50,39$ & $27,42 \pm 17,21$ & $38,41 \pm 33,20$ \\
\hline \multicolumn{4}{c}{ Plantio consorciado } \\
\hline Prof. (cm) & Areia (\%) & Silte (\%) & Argila (\%) \\
\hline $0-20$ & $12,94 \pm 2,27$ & $39,64 \pm 2,63$ & $47,42 \pm 3,96$ \\
$20-40$ & $12,19 \pm 2,25$ & $39,31 \pm 1,74$ & $48,49 \pm 3,34$ \\
\hline
\end{tabular}

Tabela 4. Teores de nutrientes comparados pelo teste $\mathrm{t}$ com a média de referência, determinada antes do plantio dos eucaliptos em substrato de cava de extração de argila, em sistemas puros e consorciados com Sesbania, aos 12 meses de idade.

Table 4. Nutrient contents compared by the $\mathrm{t}$ test with the reference average, determined before eucalyptus planting in substratum of clay mining digging, in pure and mixed systems associated with Sesbania, at 12 months after the planting.

\begin{tabular}{|c|c|c|c|c|c|c|c|c|c|}
\hline \multirow{2}{*}{ Espécie } & \multirow{2}{*}{$\begin{array}{l}\text { Prof. } \\
\text { (cm) }\end{array}$} & Consórcio & Puro & Consórcio & Puro & Consórcio & Puro & Consórcio & Puro \\
\hline & & \multicolumn{2}{|c|}{$\mathbf{P}\left(\mathrm{mg.dm}^{-3}\right)$} & \multicolumn{2}{|c|}{$\mathrm{K}\left(\mathrm{cmol}_{\mathrm{c} \cdot} \cdot \mathrm{dm}^{-3}\right)$} & \multicolumn{2}{|c|}{$\mathrm{Ca}\left(\mathrm{cmol}_{\mathrm{c}} \cdot \mathrm{dm}^{-3}\right)$} & \multicolumn{2}{|c|}{$\operatorname{Mg}\left(\mathrm{cmol}_{\mathrm{c}^{\circ}} \mathrm{dm}^{-3}\right)$} \\
\hline E. camaldulensis & $0-20$ & $15,5^{\mathrm{ns}}$ & $19,8^{\text {ns }}$ & $0,16^{\mathrm{ns}}$ & $0,14^{\mathrm{ns}}$ & $5,87^{*}$ & $6,67^{\text {ns }}$ & $3,62 *$ & $4,01 *$ \\
\hline CV (\%) & & 48,06 & 108,7 & 18,58 & 11,61 & 13,78 & 13,98 & 12,85 & 9,25 \\
\hline E. pellita & 0-20 & $17,8^{\text {ns }}$ & $32,17^{\mathrm{ns}}$ & $0,15^{\mathrm{ns}}$ & $0,15^{\mathrm{ns}}$ & $6,16^{\mathrm{ns}}$ & $7,29^{\mathrm{ns}}$ & $3,88^{*}$ & $3,58 *$ \\
\hline $\mathrm{CV}(\%)$ & & 58,50 & 66,80 & 15,06 & 13,13 & 24,70 & 30,12 & 16,49 & 16,95 \\
\hline E. robusta & 0-20 & $11,5^{*}$ & $26,69^{\mathrm{ns}}$ & $0,17^{\mathrm{ns}}$ & $0,16^{\mathrm{ns}}$ & $6,40 *$ & $7,46^{\mathrm{ns}}$ & $4,00^{*}$ & $3,74 *$ \\
\hline CV (\%) & & 20,41 & 126,09 & 18,38 & 16,71 & 4,98 & 38,35 & 8,21 & 8,63 \\
\hline E. tereticornis & $0-20$ & $15,8^{\mathrm{ns}}$ & $29,54^{\mathrm{ns}}$ & $0,15^{\mathrm{ns}}$ & $0,17^{\mathrm{ns}}$ & $6,55^{\mathrm{ns}}$ & $7,42^{\mathrm{ns}}$ & $4,22^{\mathrm{ns}}$ & $4,04^{\mathrm{ns}}$ \\
\hline CV (\%) & & 48,50 & 68,43 & 12,88 & 9,58 & 9,35 & 26,39 & 17,81 & 13,36 \\
\hline E. camaldulensis & $20-40$ & $16,1^{\mathrm{ns}}$ & $24,67^{\text {ns }}$ & $0,11^{\mathrm{ns}}$ & $0,12^{\text {ns }}$ & $6,11^{\mathrm{ns}}$ & $5,92^{\mathrm{ns}}$ & $4,12^{\mathrm{ns}}$ & $3,87^{\text {ns }}$ \\
\hline CV (\%) & & 104,88 & 100,46 & 11,81 & 28,28 & 18,68 & 20,39 & 14,97 & 12,44 \\
\hline E. pellita & $20-40$ & $15,3^{\text {ns }}$ & $27,70^{\mathrm{ns}}$ & $0,11^{\mathrm{ns}}$ & $0,11^{\mathrm{ns}}$ & $6,02^{\mathrm{ns}}$ & $6,46^{\mathrm{ns}}$ & $4,18^{\mathrm{ns}}$ & $3,36^{\mathrm{ns}}$ \\
\hline CV (\%) & & 51,97 & 67,44 & 9,57 & 16,55 & 9,55 & 41,99 & 9,03 & 13,17 \\
\hline E. robusta & $20-40$ & $10,8^{*}$ & $30,16^{\mathrm{ns}}$ & $0,14^{\mathrm{ns}}$ & $0,11^{\mathrm{ns}}$ & $6,36^{\mathrm{ns}}$ & $5,82^{\mathrm{ns}}$ & $4,12 *$ & $3,04^{\text {ns }}$ \\
\hline $\mathrm{CV}(\%)$ & & 28,02 & 66,78 & 12,42 & 15,07 & 13,27 & 34,63 & 6,93 & 17,41 \\
\hline E. tereticornis & $20-40$ & $14,0^{\text {ns }}$ & $21,89^{\mathrm{ns}}$ & $0,11^{\mathrm{ns}}$ & $0,11^{\mathrm{ns}}$ & $6,50^{\mathrm{ns}}$ & $6,50^{\mathrm{ns}}$ & $4,12 *$ & $3,74^{\mathrm{ns}}$ \\
\hline CV (\%) & & 27,57 & 67,41 & 5,53 & 30,23 & 14,50 & 45,74 & 5,60 & 14,89 \\
\hline Média referência & $0-20$ & 18,6 & 26,38 & 0,13 & 0,15 & 7,29 & 8,04 & 4,98 & 4,76 \\
\hline CV (\%) & & 54,18 & 79,20 & 20,59 & 10,67 & 18,72 & 27,46 & 14,32 & 8,28 \\
\hline Média referência & $20-40$ & 16,8 & 13,85 & 0,12 & 0,09 & 6,42 & 5,56 & 4,64 & 3,28 \\
\hline CV (\%) & & 55,10 & 51,93 & 17,81 & 25,67 & 19,29 & 43,65 & 3,03 & 34,88 \\
\hline
\end{tabular}

* Teste t: significância ao nível de $5 \% ;{ }^{\text {ns }}$ : não significativo, CV: coeficiente de variação.

Apesar de os teores de $\mathrm{Mg}$ terem diminuído em quase todos os plantios, eles continuam em níveis considerados adequados para a maioria das espécies de eucalipto cultivadas no Brasil (BARROS et al., 2000 e NOVAIS et al., 1986).

Como reflexo do observado na Tabela 4, apenas o consórcio de E. tereticornis com S. virgata não resultou na redução da capacidade de troca catiônica efetiva (CTC efetiva) e soma de bases trocáveis (SB) nos primeiros $20 \mathrm{~cm}$ de profundidade (Tabela 5). Resultados semelhantes foram encontrados por Parrota (1999), ao avaliar o desempenho de Leucaena leucocephala (Lam.) de Wit, Casuarina equisetifolia L. e Eucalyptus robusta Sm. e suas combinações. O autor verificou que, aos 7,5 anos de idade, todos os tratamentos resultaram na redução dos teores de matéria orgânica e CTC efetiva nos primeiros $40 \mathrm{~cm}$ de solo. Apesar de não terem sido constatadas diferenças entre os tratamentos, o acréscimo de $\mathrm{N}$ e $\mathrm{P}$ no solo, via serapilheira, foi menor nas parcelas que continham eucalipto consorciado com casuarina. 
Ao contrário dos resultados de Parrota (1999), os teores de matéria orgânica (MO) no substrato da cava, após um ano da implantação dos eucaliptos, não decresceram em nenhum tratamento, tendo aumentado no consórcio de $S$. virgata com E. camaldulensis, nos primeiros $20 \mathrm{~cm}$ de profundidade (Tabela 5).

Tabela 5. Características químicas comparadas pelo teste t com a média de referência, determinada antes do plantio dos eucaliptos em substrato de cava, em sistemas puros e consorciados com Sesbania, aos 12 meses de idade.

Table 5. Chemical characteristics compared by the test $\mathrm{t}$ with the reference average, determined before eucalyptus planting in substratum of clay mining digging, in pure and mixed systems associated with Sesbania, at 12 months after the planting.

\begin{tabular}{|c|c|c|c|c|c|c|c|c|c|}
\hline \multirow{2}{*}{ Espécie } & \multirow{2}{*}{$\begin{array}{l}\text { Prof. } \\
\text { (cm) }\end{array}$} & Consórcio & Puro & Consórcio & Puro & Consórcio & Puro & Consórcio & Puro \\
\hline & & \multicolumn{2}{|c|}{$\mathrm{pH}\left(\mathrm{H}_{2} \mathrm{O}\right)$} & \multicolumn{2}{|c|}{ MO $\left(\mathrm{g} \mathrm{dm}^{-3}\right)$} & \multicolumn{2}{|c|}{$t\left(\mathrm{cmol}_{\mathrm{c} .} \mathrm{dm}^{-3}\right)$} & \multicolumn{2}{|c|}{ SB $\left(\mathrm{cmol}_{\left.\mathrm{c} \cdot \mathrm{dm}^{-3}\right)}\right.$} \\
\hline E. camaldulensis & $0-20$ & $5,8^{\mathrm{ns}}$ & $6,0^{\mathrm{ns}}$ & $26,3^{*}$ & $31,3^{\text {ns }}$ & $11,0^{*}$ & $11,8^{\mathrm{ns}}$ & $10,8^{*}$ & $11,7^{\mathrm{ns}}$ \\
\hline CV (\%) & & 2,85 & 3,19 & 3,74 & 16,72 & 4,94 & 12,93 & 5,34 & 12,82 \\
\hline E. pellita & $0-20$ & $5,8^{\text {ns }}$ & $6,1^{\text {ns }}$ & $29,3^{\text {ns }}$ & $31,7^{\mathrm{ns}}$ & $11,3 *$ & $12,3^{\mathrm{ns}}$ & $11,3^{*}$ & $12,3^{\mathrm{s}}$ \\
\hline CV (\%) & & 5,31 & 7,37 & 16,82 & 36,34 & 12,33 & 11,51 & 12,25 & 11,75 \\
\hline E. robusta & $0-20$ & $5,9 *$ & $6,1^{\mathrm{ns}}$ & $26,6^{\mathrm{ns}}$ & $29,3^{\text {ns }}$ & $11,6^{*}$ & $12,5^{\mathrm{ns}}$ & $11,5^{*}$ & $12,5^{\mathrm{ns}}$ \\
\hline CV (\%) & & 7,61 & 9,55 & 10,84 & 25,61 & 4,70 & 18,72 & 4,83 & 19,15 \\
\hline E. tereticornis & $0-20$ & $5,9^{*}$ & $6,1^{\mathrm{ns}}$ & $27,7^{\mathrm{ns}}$ & $29,3^{\text {ns }}$ & $12,4^{\mathrm{ns}}$ & $12,8^{\mathrm{ns}}$ & $12,4^{\mathrm{ns}}$ & $12,7^{\mathrm{ns}}$ \\
\hline CV (\%) & & 2,11 & 6,54 & 21,36 & 17,99 & 10,02 & 17,76 & 9,94 & 18,0 \\
\hline E. camaldulensis & $20-40$ & $5,8^{\text {ns }}$ & $5,8^{\mathrm{ns}}$ & $23,8^{\text {ns }}$ & $27,6^{\text {ns }}$ & $12,6^{\mathrm{ns}}$ & $11,4^{\mathrm{ns}}$ & $12,5^{\mathrm{ns}}$ & $11,3^{\text {ns }}$ \\
\hline CV (\%) & & 3,99 & 4,25 & 34,38 & 33,59 & 9,29 & 10,63 & 9,40 & 10,93 \\
\hline E. pellita & $20-40$ & $5,8^{\mathrm{ns}}$ & $5,9^{\mathrm{ns}}$ & $22,2^{\text {ns }}$ & $20,4^{\mathrm{ns}}$ & $12,6^{\mathrm{ns}}$ & $11,9^{\text {ns }}$ & $12,5^{\mathrm{ns}}$ & $11,9^{\text {ns }}$ \\
\hline CV $(\%)$ & & 2,01 & 9,36 & 39,04 & 34,60 & 4,82 & 24,26 & 4,62 & 24,37 \\
\hline E. robusta & $20-40$ & $5,9^{\text {ns }}$ & $5,8^{\mathrm{ns}}$ & $24,6^{\mathrm{ns}}$ & $18,9^{\text {ns }}$ & $12,3^{\text {ns }}$ & $10,5^{\mathrm{ns}}$ & $12,2^{\mathrm{ns}}$ & $10,5^{\mathrm{ns}}$ \\
\hline $\mathrm{CV}(\%)$ & & 2,92 & 7,11 & 19,22 & 45,08 & 9,71 & 24,10 & 10,01 & 23,85 \\
\hline E. tereticornis & $20-40$ & $5,9^{\text {ns }}$ & $5,9^{\mathrm{ns}}$ & $24,1^{\mathrm{ns}}$ & $20,6^{\mathrm{ns}}$ & $13,1^{\mathrm{ns}}$ & $12,5^{\mathrm{ns}}$ & $13,0^{\mathrm{ns}}$ & $12,4^{\mathrm{ns}}$ \\
\hline CV (\%) & & 3,25 & 10,24 & 21,69 & 26,02 & 3,61 & 26,64 & 3,43 & 27,29 \\
\hline Média referência & $0-20$ & 5,7 & 5,7 & 24,1 & 25,2 & 13,9 & 14,1 & 13,9 & 14,1 \\
\hline CV (\%) & & 5,50 & 2,28 & 27,22 & 11,60 & 10,17 & 16,18 & 10,17 & 16,18 \\
\hline Média referência & $20-40$ & 5,7 & 6,1 & 22,0 & 16,0 & 13,0 & 10,6 & 13,0 & 10,6 \\
\hline CV $(\%)$ & & 3,20 & 6,77 & 19,26 & 41,84 & 9,05 & 30,62 & 9,05 & 30,62 \\
\hline
\end{tabular}

$\mathrm{O}$ pH do substrato não acompanhou a diminuição da CTC efetiva (Tabela 5). Na verdade, houve pequeno aumento nos consórcios de E. robusta e E. tereticornis com Sesbania.

A redução de $\mathrm{P}$ no consórcio de $E$. robusta $\operatorname{com} S$. virgata pode estar associada à maior produção de raízes finas dessa espécie em relação às demais, nesse sistema (SANTIAGO et al., np). Grant et al. (2001) afirmaram que o incremento do volume de raízes aumenta a habilidade da planta em acessar e absorver o $\mathrm{P}$ do solo, já que este apresenta baixa mobilidade nos solos, e a maior parte de seu transporte até a região das raízes se dá por difusão, sendo esta favorecida pela interceptação radicular.

A poda da Sesbania resultou no aporte de $2,5 \mathrm{~kg}$ de biomassa seca por planta, com elevados teores de nitrogênio (Tabela 6).

Os valores de N, P e K encontrados na biomassa resultante da poda na Sesbania foram superiores aos encontrados na mesma espécie, aos 10 meses de idade, em cavas de extração de argila, por Coutinho (2003). Mafra et al. (1998a), no cerrado, encontraram, para Leucaena leucocephala, valores de N, P e K próximos aos encontrados no presente estudo, evidenciando-se que a Sesbania, mesmo em condições de cavas de extração de argila, aporta quantidade significativa de nutrientes, via podas periódicas, mais notadamente de nitrogênio. As quantidades de N, P, K, Ca e Mg, em kg.ha ${ }^{-1}$, foram de 323,9; 38,7; 105,0; 28,0 e 6,2 , respectivamente.

Outro fator importante a ser considerado na avaliação do potencial da Sesbania como espécie adubadeira é a baixa relação $\mathrm{C}: \mathrm{N}$ das folhas (11) e frutos (13), que potencializariam a rápida disponibilização dos nutrientes contidos no material vegetal, no substrato da cava. Ao contrário, os galhos da Sesbania, com elevada relação C:N (84), evidenciam a importância desse componente da planta na manutenção dos teores de matéria orgânica no substrato, com liberação gradativa dos nutrientes ao longo do tempo. 
Tabela 6. Aporte de nutrientes, massa seca de folhas, galhos e frutos de Sesbania virgata quantificados após a poda, em consórcio com espécies de eucalipto, aos 22 meses de idade, em cavas de extração de argila.

Table 6. Input of nutrients, mass dries of Sesbania virgata leaves, branches and fruits, quantified after the pruning, in mixed plantings with eucalyptus species, 22 months after planting in clay mining diggings.

\begin{tabular}{lcccccccccccc}
\hline \multirow{2}{*}{ Parte } & $\begin{array}{l}\text { Massa seca } \\
\left.\text { (g.planta }^{-1}\right)\end{array}$ & $\mathbf{N}$ & $\mathbf{P}$ & $\mathbf{6}$ & $\mathbf{C a}$ & $\mathbf{M g}$ & $\mathbf{S}$ & $\mathbf{C}$ & $\mathbf{F e}$ & $\mathbf{C u}$ & $\mathbf{Z n}$ & $\mathbf{M n}$ \\
\hline Folha & 654,9 & 37,9 & 2,9 & 10,8 & 4,2 & 0,7 & 3,2 & 397,4 & 162,7 & 4,3 & 30,4 & 100,2 \\
CV $(\%)$ & 29,33 & 9,82 & 8,48 & 12,78 & 22,15 & 16,20 & 35,01 & 68,78 & 11,03 & 11,62 & 11,58 & 31,46 \\
Galho & 1394,5 & 8,8 & 1,9 & 8,8 & 1,0 & 0,3 & 1,3 & 439,6 & 61,1 & 12,0 & 34,1 & 13,3 \\
CV (\%) & 39,36 & 10,34 & 13,24 & 22,77 & 19,60 & 14,08 & 58,01 & 5,03 & 32,97 & 119,83 & 15,64 & 28,39 \\
Fruto & 391,3 & 32,9 & 4,7 & 6,2 & 1,7 & 0,5 & 2,6 & 405,9 & 184,8 & 5,9 & 34,7 & 34,6 \\
CV (\%) & 59,50 & 7,01 & 19,65 & 26,15 & 17,09 & 14,47 & 55,23 & 62,26 & 60,96 & 50,17 & 20,90 & 36,08 \\
\hline
\end{tabular}

$\mathrm{CV}$ : coeficiente de variação.

\section{CONCLUSÕES}

- Os teores de matéria orgânica não decresceram em nenhum dos plantios.

- De modo geral, os plantios consorciados reduziram de maneira mais acentuada os teores de soma de bases (SB) e capacidade de troca catiônica efetiva $(\mathrm{t})$ presentes no substrato da cava em relação aos monocultivos, possivelmente em função da maior quantidade de plantas por unidade de área (eucalipto + Sesbania).

- O plantio consorciado de E. robusta com S. virgata foi o tratamento que mais reduziu os teores de nutrientes avaliados.

- Os plantios com E. tereticornis, em monocultivo ou consorciados, não alteraram os teores de nutrientes, a capacidade de troca catiônica efetiva e a SB no substrato, no primeiro ano após o plantio.

\section{REFERÊNCIAS}

BARROS, N. F.; NEVES, J. C. L.; NOVAIS, R. F. Recomendação de fertilizantes minerais em plantios de eucalipto. In: GONÇALVES, J. L. M.; BENEDETTI, V. (Eds.). Nutrição e Fertilização Florestal. Piracicaba, SP: IPEF, 2000, p. 269-286.

CAMPOS, H. Estatística Experimental Não Paramétrica. 3 ed. Piracicaba: ESALQ/USP, 1979. 373 p.

COUTINHO, M. P. Crescimento de Sesbania virgata (Cav.) Pers. em cavas de extração de argila. 54 p. Dissertação (Mestrado em Produção Vegetal) - Universidade Estadual do Norte Fluminense, Campos dos Goytacazes, RJ, 2003.

DIONÍSIO, J. A.; TANCK, B. C. B.; dos SANTOS, A.; SILVEIRA, V. I.; dos SANTOS, H. R. Avaliação da população de oligochaeta (terrestres) em áreas degradadas. Revista do Setor de Ciências Agrárias, Curitiba, v. 13, n. (1-2), p. 35-40, 1994.

EMPRESA BRASILEIRA DE PESQUISA AGROPECUÁRIA. Centro Nacional de Pesquisa do Solo (EMBRAPA.CNPS). Manual de Métodos de Análise de Solo. $2^{\text {a }}$ ed. rev. atual. Rio de Janeiro, 1997. 212 p. (Documentos, n 1).

FREITAS, T. A. S. de. Sistema de blocos prensados para a produção de mudas de eucalipto. 92 p. Dissertação (Mestrado em Produção Vegetal) - Universidade Estadual do Norte Fluminense, Campos dos Goytacazes, RJ, 2003.

GACHENGO, C. N.; PALM, C. A.; JAMA, B.; OTHIENO, C. Tithonia and senna green manures and inorganic fertilizers as phosphorus sources for maize in Western Kenya. Agroforestry Systems, Dordrecht, n. 44, p. 21-36, 1999.

GRANT, C. A.; FLATEN, D. N.; TOMASIEWICZ, D. J.; SHEPPARD, S. C. A importância do fósforo no desenvolvimento inicial da planta. Informações Agronômicas, Piracicaba, n. 95. 2001 
HAGGAR, J. P.; TANNER, E. V. J.; BEER, J. W.; KASS, D. C. L. Nitrogen dynamics of tropical agro forestry and annual cropping systems. Soil Biology and Biochemistry, Elmsford, v. 25, n. 10, p. 13631378, 1993.

JONES Jr., J. B.; WOLF, B.; MILLS, H. A. Plant analysis handbook: a practical sampling, preparation, analysis and interpretation guide. Athens (USA): Micro - Macro Publishing, 1991. 213 p.

KIEHL, E. J. Manual de Edafologia. São Paulo: Ceres, 1979. 262 p.

KUMAR, B. M. Intercropping teat with Leucena increases tree growth and modifies soil characteristics. Agroforest Systems, Dordrecht, n. 42, p. 81-89, 1998.

MAFRA, A. L.; MIKLÓS, A. A. W. de; VOCURCA, H. L.; HARKALY, A. H.; MENDONZA, E. Produção de fitomassa e atributos químicos do solo sob cultivo em aléias e sob vegetação nativa de cerrado. Revista Brasileira de Ciência do Solo, Campinas, n. 22, p. 43-48, 1998a.

MAFRA, A. L.; MIKLÓS, A. A. W. de; VOCURCA, H. L.; HARKALY, A. H.; MENDONZA, E. Adição de nutrientes ao solo em sistema agroflorestal do tipo "cultivo em aléias" e em cerrado na região de Botucatu, SP. Scientia Forestalis, Piracicaba, n. 54, p. 41-54, 1998b.

Malavolta, E.; VITTI, G. C.; OliVeIRA, S. A. Avaliação do Estado Nutricional das Plantas, Princípios e Aplicações. 2. ed. Piracicaba: Potafo, 1997. 319 p.

MARTINS, S. G.; SILVA, M. L. N.; CURI, N.; FERREIRA, M. M. Avaliação de atributos físicos de um LATOSSOLO DISTROFÉRRICO sob diferentes povoamentos florestais. Cerne, Lavras, v.8, n. 1, p. 3241, 2002.

MORAES, M. H.; MÜLLER, M. M. L.; FOLONI, J. S. S. Qualidade física do solo: métodos de estudo sistema de preparo e manejo do solo. Jaboticabal, SP: Funep, 2002. np.

NOVAIS, R. F.; BARROS, N. F.; NEVES, J. C. L. Interpretação de análise química do solo para o crescimento e desenvolvimento de Eucalyptus spp. Níveis críticos de implantação e de manutenção. R. Árvore, Viçosa, MG, v. 10, n.1, p. 105-111, 1986.

PARROTA, J. A. Productivity, nutrient cycling, and succession in single- and mixed-species plantations of Casuarina equisetifolia, Eucalyptus robusta and Leucaena leucocephala in Puerto Rico, Forest Ecology and Management, Amsterdam, v. 124, n. 1, p. 45-77, 1999.

RUIVO, M. L. P.; BARROS, N. F.; SCHAEFFER, C. E. R. Vegetação, biomassa microbiana e características químicas do solo como indicadores de reabilitação de áreas mineradas na Amazônia Oriental. Revista de Ciências Agrárias, Belém, n. 36, p. 137-160, 2001.

SANTIAGO, A. R.; BARROSO, D. G.; MENDONÇA, A. V. R.; CAMPOSTRINI, E.; DETMANN, E.; CARNEIRO, J. G. A. Crescimento inicial de eucalipto em cavas de extração de argila, sob dois sistemas de cultivo. (dados não publicados).

SOUZA, P. A.; VENTURIN, N.; MACEDO, R. L. G.; ALVARENGA, M. I. N.; SILVA, V. F. Estabelecimento de espécies arbóreas em recuperação de área degradada pela extração de areia. Cerne, Lavras, v. 7, n.2, p. 43-52, 2001.

VALICHESKI, R. R.; SCHIAVO, J. A.; MARCIANO, C. R.; MARTINS, M. A.; RODRIGUES, L. A.; MENDONÇA, A. V. R. Atributos físicos do substrato remanescente da extração de argila no município de Campos dos Goytacazes, RJ. In.: FERTIBIO 2003, Ribeirão Preto. Anais... Ribeirão Preto: SBCS, 2003. (CD-ROM).

VALICHESKI, R. R. Avaliação técnica e econômica de cenários de uso da terra em áreas degradadas pela atividade de extração de argila em campos dos Goytacazes, RJ. 138 p. Dissertação (Mestrado em Produção Vegetal) - Universidade Estadual do Norte Fluminense, Campos dos Goytacazes, RJ, 2004.

VEZZANI, F. M.; TEDESCO, M. J.; BARROS, N. F. Alterações dos nutrientes no solo e nas plantas em consórcio de eucalipto e acácia negra. Revista Brasileira de Ciência do Solo, Campinas, n. 25, p. 22523, 2001. 